\title{
Conditioned Cancellation Modules
}

Bothaynah N. Shihab,

Department of Mathematics, College of Education for Pure Science/Ibn-Al-Haitham, University of Baghdad.

\section{Introduction 1}

Let $\mathrm{R}$ be a commutative ring and $\mathrm{M}$ a unital $\mathrm{R}$-module. Gilmer [1, $\mathrm{p} .60]$ has been defined the concept of cancellation ideal to be the ideal I of $\mathrm{R}$ which satisfies the following:-

Whenever $\mathrm{AI}=\mathrm{BI}$ with $\mathrm{A}$ and $\mathrm{B}$ are ideals of $\mathrm{R}$ implies $\mathrm{A}=\mathrm{B}$

Mijbass in [2] has been generalized this concept to modules. He has been defined the cancellation modules as follows:

An R-module $\mathrm{M}$ is called a cancellation module whenever $\mathrm{AM}=\mathrm{BM}$ with $\mathrm{A}$ and $\mathrm{B}$ are ideals of $\mathrm{R}$ implies $\mathrm{A}=\mathrm{B}$.

In this work we shall introduce the concept of semiprime cancellation module by using some restrictions on the ideals A and B in the above definition, namely we shall say that

An $\mathrm{R}$-module $\mathrm{M}$ is called semiprime cancellation, whenever $\mathrm{AM}=\mathrm{BM}$ with $\mathrm{A}$ is $\mathrm{a}$ semiprime ideal of $\mathrm{R}$ and $\mathrm{B}$ is any ideal of $\mathrm{R}$ implies $\mathrm{A}=\mathrm{B}$.

An ideal $\mathrm{A}$ of a ring $\mathrm{R}$ is said to be semiprime if $\mathrm{A} \cap \mathrm{B}=\mathrm{BA}$ for all ideal $\mathrm{B}$ of $\mathrm{R}$.

Clearly, the class of cancellation modules contains the class of semiprime cancellation modules and we can give an example to show that this inclusion is properly. However we shall give conditions under which the two classes are equivalent, see proposition (1.9).

This paper consists of two sections our principal aim in the

first section is to study the semiprime cancellation modules. Moreover we study the relationships between cancellation modules and semiprime cancellation module. Also, we discuss the property of semiprime cancellation in each of the module and its trace, where we prove that a module is semiprime cancellation if its trace is a semiprime cancellation ideal, see corollary (1.12). 


\section{Semiprime Cancellation Modules2}

In this section we introduce the concept of semiprime cancellation modules with some examples and basic properties about this concept. Also, we investigate semiprime cancellation module by using the trace of the module. Finally, we study the relation between semiprime cancellation modules and some types of modules.

\section{Definition (2.1):-}

An $\mathrm{R}$-module $\mathrm{M}$ is called semiprime cancellation whenever $\mathrm{AM}=\mathrm{BM}$, with $\mathrm{A}$ is $\mathrm{a}$ semiprime ideal of $R$ and $B$ is any ideal of $R$, implies $A=B$.

\section{Examples and Remarks (2.2):-}

(1) $Z_{6}$ as a $Z_{12}$-module is semiprime cancellation module

It is clear that (3) is pure ideal of $\mathrm{Z}_{12}$ and (3) $\mathrm{Z}_{6}=(9) \mathrm{Z}_{6}$. Then (3) $=(9)$.

(2) Every cancellation module is semiprime cancellation module, but the converse is not true in general, for example: $Z_{6}$ as a $Z_{12}$-module. See above example number 1 . But $Z_{6}$ is not cancellation $Z_{12}$-module, since an $n R\left(Z_{6}\right)=(6)$ is not faithful and hence $Z_{6}$ is not cancellation by [2, remark (1-4), p.8].

(4) $Z_{5}$ as a $Z_{15}$-module is semiprime cancellation module. Since, (3) is semiprime ideal of $Z_{15}$ and (3) $Z_{5}=(6) Z_{5}$. Then (3) = (6) .Also, (5) is semiprime ideal of $Z_{15}$ and (5) $Z_{5}=(10) Z_{5}$.

Then $(5)=(10)$.

Recall that the element $\mathrm{m}$ in an R-module $\mathrm{M}$ (where $\mathrm{R}$ is an integral domain) is called torsion element if there exists $0 \neq \mathrm{r} \in \mathrm{R}$ such that $\mathrm{rm}=0$. And $\mathrm{m}$ is called a non-torsion element if $\mathrm{rm}$ $\neq 0, \forall 0 \neq \mathrm{r} \in \mathrm{R},[2]$

For cyclic modules we have the following result:-.

Proposition (2.3):- Every cyclic module generated by a non-torsion element is semiprime cancellation .

Proof: Let $\mathrm{M}=\langle\mathrm{m}\rangle$, where $\mathrm{m}$ is a non-torsion element and $\mathrm{A}\langle\mathrm{m}\rangle=\mathrm{B}\langle\mathrm{m}\rangle$, where $\mathrm{A}$ is pure ideal of $R$ and $B$ is any ideal of $R$. $a m \in B<m>$ for all $a \in A$, then $a m=b m$,

where $b \in B$, implies $a m-b m=0$. Therefore $(a-b) m=0$, but $m$ is a non-torsion element, then $\mathrm{a}-\mathrm{b}=0$, which implies $\mathrm{a}=\mathrm{b}$. Therefore $\mathrm{A} \subseteq \mathrm{B}$.

Similarly $\mathrm{B} \subseteq \mathrm{A}$, and hence $\mathrm{A}=\mathrm{B}$.

We shall show by an example that the condition $\mathrm{M}$ is generated by a non-torsion element in proposition (2.3) can not be dropped.

Example (2.4):-

Let $\mathrm{M}=\mathrm{Z}_{2}$ as a $\mathrm{Z}$-module, it is clear that $\mathrm{Z}_{2}=(1)$ and

is a torsion element in $Z_{2}$ and $Z_{2}$ is not semiprime cancellation $Z_{4}$-module.

In the following theorem we give some characterizations of semiprime cancellation modules.

Theorem (2.5) :- Let M be an R-module. Then the following statements are equivalent:-

(1) $\mathrm{M}$ is semiprime cancellation module.

(2) If $\mathrm{AM} \subseteq \mathrm{BM}$, such that $\mathrm{A}$ is any ideal of $\mathrm{R}$ and $\mathrm{B}$ is a semiprime ideal of $\mathrm{R}$, then $\mathrm{A} \subseteq \mathrm{B}$. 
(3) If $\langle\mathrm{a}>\mathrm{M} \subseteq \mathrm{BM}$, such that $\mathrm{a} \in \mathrm{R}$ and $\mathrm{B}$ is a semiprime ideal of $\mathrm{R}$, then $\mathrm{a} \in \mathrm{B}$

. (4) $(\mathrm{AM}: \mathrm{M})=\mathrm{A}$ for all semiprime ideals $\mathrm{A}$ of $\mathrm{R}$

(5) $(\mathrm{AM}: \mathrm{BM})=(\mathrm{A}: \mathrm{B})$, for all ideals $\mathrm{B}$ of $\mathrm{R}$ and for all semiprime ideals $\mathrm{A}$ of $\mathrm{R}$

Proof: (1) $\Rightarrow$ (2) suppose that $\mathrm{M}$ is semiprime cancellation module and $\mathrm{AM} \subseteq \mathrm{BM}$, where

$B$ is a semiprime ideal of $R$ and $A$ is any ideal of $R$. Now, $B M=A M+B M=(A+B) M$, then $\mathrm{B}=\mathrm{A}+\mathrm{B}$ implies $\mathrm{A} \subseteq \mathrm{B}$.

(2) $\Rightarrow$ (3) Let $\langle a>M \subseteq B M$. Then $\langle a\rangle \subseteq B$ by (2). Hence $a \in B$.

(3) $\Rightarrow$ (4) Let $\mathrm{x} \in(\mathrm{AM}: \mathrm{M})$. Then $\mathrm{xM} \subseteq \mathrm{AM}$ by (3) $\mathrm{x} \in \mathrm{A}$. Hence $(\mathrm{AM}: \mathrm{M}) \subseteq \mathrm{A}$.

On the other side if $x \in A$, then $x M \subseteq A M$. Therefore $x \in(A M: M)$ and hence $(A M: M)=A$.

$4) \Rightarrow(5)$ Let $x \in(A M: M)$. Then $x \in((A M: M), B)$ since $(A M: M)=A($

by (4), implies $x \in(\mathrm{AM}: \mathrm{BM})$ [4,proposition (2.3),p.38]

Now if $\mathrm{x} \in(\mathrm{AM}: \mathrm{BM})=((\mathrm{AM}: \mathrm{M}): \mathrm{B})$ and since $(\mathrm{AM}: \mathrm{M})=\mathrm{A}$ by $(4)$.

) $j$ Then $x \in(A: B)$. Therefore $(A M: B M)=(A: B$

$(5) \Rightarrow(1)$ Let $A M=B M$ and $A$ is a semiprime ideal of $R, B$ is any ideal of $R$. Then $(\mathrm{AM}: \mathrm{BM})=\mathrm{R}$ implies $(\mathrm{A}: \mathrm{B})=\mathrm{R}$. Therefore $\mathrm{B} \subseteq \mathrm{A}$.

Similarly $\mathrm{A} \subseteq \mathrm{B}$. Then $\mathrm{A}=\mathrm{B}$. Hence $\mathrm{M}$ is semiprime cancellation module

In order to give another characterization of semiprime cancellation module we need to recall the definition of tensor product of modules and some related lemma: A tensor product of two R-modules $A$ and $B$ means a pair $(M, T)$ where $M$ is an R-module and $T: A \times B \rightarrow M$ is a bilinear map, such that for any $\mathrm{R}$-module $\mathrm{X}$ and any bilinear map $\mathrm{g}$ : $\mathrm{A} \times \mathrm{B} \rightarrow \mathrm{X}$, there exists a unique homeomorphism $\mathrm{h}: \mathrm{M} \rightarrow \mathrm{X}$

such that hoT $=\mathrm{g}[3]$.

The following proposition gives a necessary and sufficient condition for a module to be semiprime cancellation.

\section{Proposition (2.6) :-}

Let $\mathrm{M}$ be an R-module. Then $\mathrm{M}$ is semiprime cancellation module if and only if $\operatorname{ann}_{R}(\mathrm{M} \otimes R)$ $=\mathrm{A}$ for all semiprime ideals $\mathrm{A}$ of $\mathrm{R}$.

Proof: Let $\mathrm{M}$ be semiprime cancellation module. And by [2, proposition (1.11), p.12], we get $\mathrm{M} \otimes \mathrm{R} / \mathrm{A} \cong \mathrm{M} / \mathrm{AM}$, where $\mathrm{A}$ is semiprime ideal of $\mathrm{R}$. Therefore $\operatorname{ann}_{\mathrm{R}}(\mathrm{M} \otimes \mathrm{R} / \mathrm{A})=$

$(\mathrm{AM}: \mathrm{M})$ $=\mathrm{A}$ by theorem $(1.5,(4))$

Now suppose $\operatorname{ann}_{R}(M \otimes R / A)=A$ for all semiprime ideal $A$ of $R . M \otimes R / A \cong M / A M$ by [2, proposition (1.11), p.12]. Then $\operatorname{ann}_{R}(M / A M)=\operatorname{ann}_{R}(M \otimes R / A)=A$. But $\operatorname{ann}_{R}(M / A M)=$ $(\mathrm{AM}: \mathrm{M})$. Therefore $(\mathrm{AM}: \mathrm{M})=\mathrm{A}, \mathrm{M}$ is semiprime cancellation module by theorem $(1.5,(4))($

\section{Proposition (2.7):-}

If $\mathrm{M}$ is an $\mathrm{R}$-module and $\mathrm{N}$ is a homeomorphic image of $\mathrm{M}$, which is semiprime cancellation R-module, then $\mathrm{M}$ is semiprime cancellation module.

Proof: Let $A M=B M$, where $A$ is pure ideal of $R$ and $B$ is any ideal of $R$. And let $\varnothing: M \rightarrow N$ be an epimorphism such that $N$ is semiprime cancellation module. Then $\varnothing(A M)=\varnothing(B M)$. But $\varnothing(\mathrm{AM})=\mathrm{A} \varnothing(\mathrm{M})=\varnothing(\mathrm{BM})=\mathrm{B} \varnothing(\mathrm{M})$. Then $\mathrm{AN}=\mathrm{BN}$. But

$\mathrm{N}$ is semiprime cancellation $\mathrm{R}$-module. Then $\mathrm{A}=\mathrm{B}$ which completes the proof. 
From proposition (1.7), we get the following result :-

\section{Corollary (2.8) :-}

If $\mathrm{M}$ has a semiprime direct summand, then $\mathrm{M}$ is also semiprime cancellation R-module.

\section{Trace of Semi Cancellation Modules}

In this section we study the trace of semiprime cancellation module. Some results about this study is submitted.

The following proposition gives a condition under which the property of semiprime cancellation and cancellation are equivalents.

\section{Proposition (3.1) :-}

Let $\mathrm{M}$ be an $\mathrm{R}$-module. Then $\mathrm{M}$ is a cancellation module if and only if $\mathrm{M}$ is faithful semiprime cancellation module.

Proof: It is know that, every cancellation module is semiprime cancellation module, and every cancellation module is faithful module, [2, remark (1.4), p.8]

Conversely: suppose that $\mathrm{M}$ is faithful semiprime cancellation module. Let $\mathrm{AM}=\mathrm{BM}$, where $\mathrm{A}$ and $\mathrm{B}$ are two ideals in $\mathrm{R}$.

If $\mathrm{A}$ is pure ideal of $\mathrm{R}$ and $\mathrm{B}$ is any ideal of $\mathrm{R}$, implies $\mathrm{A}=\mathrm{B}$ (since $\mathrm{M}$ is semiprime cancellation module)

If $A$ is not semiprime ideal of $R$ and $B$ is any ideal of $R, A M-B M=0 \Rightarrow A-B \subseteq \operatorname{ann}(M)=$ 0 . Hence $\mathrm{A}=\mathrm{B}$.

Now, we have the following.

\section{Proposition (3.2) :-}

Let $\mathrm{M}$ be a cancellation $\mathrm{R}$-module and $\mathrm{A}$ be an ideal in $\mathrm{R}$. Then $\mathrm{AM}$ is semiprime cancellation module if and only if $\mathrm{A}$ is semiprime cancellation ideal.

Proof: Suppose that AM is semiprime cancellation module. To prove that $\mathrm{A}$ is semiprime cancellation ideal. Let $\mathrm{BA}=\mathrm{CA}$, where $\mathrm{B}$ is a pure ideal of $\mathrm{R}$ and $\mathrm{C}$ is any ideal of $\mathrm{R}$. Since, $\mathrm{BAM}=\mathrm{CAM}$, implies $\mathrm{C}=\mathrm{B}$. Therefore $\mathrm{A}$ is semiprime cancellation ideal.

Conversely, suppose that $\mathrm{A}$ is semiprime cancellation ideal and BAM $=\mathrm{CAM}$, where $\mathrm{B}$ is semiprime ideal of $\mathrm{R}$ and $\mathrm{C}$ is any ideal of $\mathrm{R}$. Then $\mathrm{BA}=\mathrm{CA}$ (since $\mathrm{M}$ is semiprime cancellation module), implies $\mathrm{B}=\mathrm{C}$ (since $\mathrm{A}$ is semiprime cancellation ideal). Therefore AM is semiprime cancellation module.

\section{Proposition (3.3) :-}

Let $\mathrm{M}$ and $\mathrm{N}$ be two R-modules and $\mathrm{L}=\sum \emptyset_{\alpha}(\mathrm{M})$ be a submodule of $\mathrm{N}$, where the sum is taken for any subset of $\operatorname{Hom}(\mathrm{M}, \mathrm{N})$, such that $\mathrm{L}$ is semiprime cancellation module. Then $\mathrm{M}$ is semiprime cancellation module.

Proof: Let $\mathrm{AM}=\mathrm{BM}$, where $\mathrm{A}$ is a semiprime ideal of $\mathrm{R}$ and $\mathrm{B}$ is any ideal of $\mathrm{R}$. Then $\emptyset_{\alpha}(\mathrm{AM})=\emptyset_{\alpha}(\mathrm{BM})$, for each $\emptyset_{\alpha} \in \operatorname{Hom}(\mathrm{M}, \mathrm{N})$

implies

$\sum_{\emptyset_{\alpha} \in H o m(M, N)} \emptyset_{\alpha \alpha}(A M)=\sum_{\emptyset_{\alpha} \in H o m(M, N)} \emptyset_{\alpha}(B M)$.But $\emptyset_{\alpha}(A M)=A \emptyset_{\alpha}(M)=B \emptyset_{\alpha}(M)$ .Then $\mathrm{A} \sum_{\emptyset_{\alpha} \in H o m(M, N)} \emptyset_{\propto}(M)=B \sum_{\emptyset_{\alpha} H o m(M, N)} \emptyset_{\propto}(M)$. 
Therefore $\mathrm{AL}=\mathrm{BL}$, implies $\mathrm{A}=\mathrm{B}$ (since $\mathrm{L}$ is semiprime cancellation submodule)

\section{Corollary (3.4) :-}

If $\mathrm{M}$ is an $\mathrm{R}$-module and $\mathrm{T}(\mathrm{M})$ is semiprime cancellation ideal of $\mathrm{R}$, then $\mathrm{M}$ is semiprime cancellation module.

Proof: The result is clear by using the definition of $\mathrm{T}(\mathrm{M})$ and proposition (3.3) .

\section{Corollary (3.5) :-}

If $\mathrm{M}$ is an $\mathrm{R}$-module and $\mathrm{T}(\mathrm{M})$ is multiplication ideal of $\mathrm{R}$, which contain a non-zero divisor element, then $\mathrm{M}$ is semiprime cancellation module.

Proof: Let $\mathrm{a} \in \mathrm{T}(\mathrm{M})$ and $\mathrm{a}$ is a non-zero divisor. $\mathrm{T}(\mathrm{M})$ is a multiplication ideal of $\mathrm{R}$, so there exists an ideal $\mathbf{J}$ of $\mathrm{R}$, such that: $\langle\mathrm{a}\rangle=\mathrm{JT}(\mathrm{M})$. Implies $\mathrm{T}(\mathrm{M})$ is an invertable ideal of $\mathrm{R}$ [4,proposition (6.3), p.125]. Therefore $\mathrm{T}(\mathrm{M})$ is a cancellation ideal [5, p.879]. Implies $\mathrm{T}(\mathrm{M})$ is semiprime cancellation module. Then $\mathrm{M}$ is semiprime cancellation module by corollary (1.12).

\section{Corollary (3.6) :-}

Let $\mathrm{M}$ be an R-module, such that $\mathrm{T}(\mathrm{M})$ is semiprime cancellation ideal. Then $\mathrm{M}^{*}=$ $\operatorname{Hom}(\mathrm{M}, \mathrm{R})$ is semiprime cancellation R-module.

Proof: Let aM* $\subseteq \mathrm{BM}^{*}$, such that $\mathrm{B}$ is a semiprime ideal of $\mathrm{R}$.

Now, a $\mathrm{f} \in \mathrm{aM}^{*} \subseteq \mathrm{BM}^{*}, \forall \mathrm{f} \in \mathrm{M}^{*}$. Thus a $\mathrm{f} \in \mathrm{BM}^{*}$, implies

a f $=\sum_{i=1}^{n} b_{i} f_{i}(m)$, where bi $\in \mathrm{B}$ and fi $\in \mathrm{M}^{*}, \forall \mathrm{m} \in \mathrm{M}$.

But $\mathrm{T}(\mathrm{M})$ is semiprime cancellation ideal. Then $\mathrm{a} \in \mathrm{B}$ by theorem $((2.3),(3))$ and hence $\mathrm{M}^{*}$ is semiprime cancellation module.

Recall that a ring $\mathrm{R}$ is called regular (Von-Numann) if for each element $\mathrm{a} \in \mathrm{R}$, there exists an element $r \in R$ such that a

$$
\text { a } r \text { a ( } a=a 2 r \text { if } R \text { is commutative), [6 ] }
$$

An R-module $\mathrm{M}$ is flat if for each injective homomorphism f: $\bar{N} \rightarrow \mathrm{N}$ from one R-module into the homomorphism $1_{\mathrm{M}} \otimes \mathrm{f}: \mathrm{M} \otimes \mathrm{N}^{\prime} \rightarrow_{\mathrm{R}} \mathrm{M} \otimes \mathrm{N}$ is injective, where $1_{\mathrm{M}}$ is the identity isomorphism of $\mathrm{M},[6]$.

Now, we give the following result:-

\section{Proposition (3.7):-}

Let $\mathrm{M}$ be a module over a regular ring $\mathrm{R}$ such that $\mathrm{AM}$ is faithful for all ideal $\mathrm{A}$ of $\mathrm{R}$. Then $\mathrm{M}$ is semiprime cancellation module.

Proof: Let $\mathrm{AM}=\mathrm{BM}$, where $\mathrm{A}$ is semiprime ideal of $\mathrm{R}$ and $\mathrm{B}$ is any ideal of $\mathrm{R}$. From a ring $\mathrm{R}$ is regular by [8, proposition(1.10)].

we get $A^{2} \mathrm{M}=B^{2} \mathrm{M}$. Then $\mathrm{A}(\mathrm{AM})=\mathrm{B}(\mathrm{BM})$

which implies $\mathrm{A}(\mathrm{AM})=\mathrm{B}(\mathrm{AM})$ and hence $\mathrm{A}-\mathrm{B} \subseteq \mathrm{ann}(\mathrm{AM})=0$. (since $\mathrm{AM}$ is a faithful for all ideal $\mathrm{A}$ of $\mathrm{R})$. Thus $\mathrm{A}=\mathrm{B}$

Therefore $\mathrm{M}$ is semiprime cancellation module.

.following corollary As an application of proposition (2.15), we give the 


\section{Corollary (3.8) :-}

Let $\mathrm{M}$ be a flat $\mathrm{R}$-module and $\mathrm{AM}$ is faithful for all ideal $\mathrm{A}$ of $\mathrm{R}$. hen $\mathrm{M}$ is semiprime cancellation module .

Proof: It is obvious according to [8, proposition (1.12), p.9] and proposition (2.15).

\section{REFERENCES}

I. R.W. Gilmer, (1972), Multiplicative Ideal Theory, Marcel Dekker, New York.

II. S. Majbas, (1992), On Cancellation Modules, M.Sc. Thesis, University of Baghdad.

III. Majid M. Ali and David J. Smith, (2004), Pure Submodules of Multiplication Modules, Beiträge Zur Algebra and Geometric, Contributions to Algebra and Geometry, Vol.25, No. 1, pp.61-74.

IV. M.D. Larsen and Maccar the P.J., (1971), Multiplication Theory of Ideals, Academic Press, London, New York

V. Anderson D.D., (2000), Some Remarks on Multiplication Ideals II, Comm. In Algebra, 28.

VI. H.Y.Khalaf, (2007), Semimaximal Submodules, Ph.D.Thesis, University of Baghdad

VII. N.S. Abdul-Karim, (1993), On Z-Regulars Modules, M.Sc. Thesis, College of Science, University of Baghdad. 